\title{
Survey finds only $15 \%$ of emergency departments have formal alcohol screening and intervention policies for trauma patients Paolo Deluca
}

$10.1136 /$ ebn1001

Institute of Psychiatry, King's College London, London, UK

Correspondence to: Paolo Deluca De Crespigny Park P048, London SE5 8AF, UK; paolo.deluca@kcl.ac.uk

Commentary on: Cunningham RM, Harrison SR, McKay MP, et al. National survey of emergency department alcohol screening and intervention practices. Ann Emerg Med 2010;55:556-62.

In this study, Cunningham and colleagues sought to understand the degree to which alcohol screening and brief interventions (ASBI) are implemented across Emergency Departments (EDs) nationwide in the USA.

There already exists a substantial evidence base for the efficacy of brief interventions aimed at reducing alcohol consumption across a range of healthcare settings, ${ }^{1}$ and a previous review from the same authors highlighted how EDs offer also a unique opportunity to address the burden of alcohol misuse. ${ }^{2}$ However, ASBI implementation in ED settings has so far been patchy, even though the vast number of patients that visit ED each year, with or without alcohol-related presentations, offers many opportunities to access and intervene with a large number of patients who may misuse alcohol.

More than 14 million people are treated in Emergency Departments (ED) in England each year, of which 35\% of attendances are alcohol related. Furthermore, a survey of 32 EDs in England found that up to 40\% of admissions at weekends and up to $70 \%$ at peak times were alcohol related. ${ }^{3}$ These high levels of attendances can be explained by the link between excessive drinking and a greater risk of being involved in accidents, assaults, fights and other traumatic events requiring hospital care. ${ }^{3}$ ${ }^{4}$ Moreover, the rising patterns in alcohol consumption suggest that the prevalence of Alcohol Use Disorders
(AUD) might increase significantly over the next 10 years, which poses a significant threat to the health of the UK population. It is also clear that there is no need for further evidence that ED is a good setting to target AUD. However, we definitely need to understand how ASBI can be effectively and sustainably implemented in these challenging environments.

In this study, Cunningham and colleagues surveyed all 441 ED directors in the USA about their current alcohol screening and intervention practices, their attitudes, knowledge and perceived facilitator and/or impeding factors. Only 46\% responded and, although this is in line with previous surveys, and that there were no apparent differences between responders and non-responders, one might still argue that figures and findings in reality might look even worse than those reported. This might also reflect the fact that ASBI is not on top of the agenda for many ED directors.

Indeed, the authors reported that even though many US agencies and organisations promote and encourage alcohol screening and brief interventions in ED, these were not yet widely implemented. More worryingly, where these were implemented it was neither done in a systematic way, nor with the use of preferred screening tools. Indeed, breathalyzer was the most common screening method reported (64.5\%) even though faster and cheaper 
tools exist (eg, FAST, SASQ, AUDIT). Questionnaire methods of screening are considerably more valid and cost-effective than blood-screening methods. ${ }^{5}$

Moreover, once the patients were identified the intervention they received was not a standardised intervention in the form of admonishment; caution provided by the physician. Only $9 \%$ reported the provision of brief alcohol intervention by personnel who have received formal training.

Overall, ED directors seemed to have a positive attitude, recognise the size of the problem and agree that extra and dedicated support in ED would benefit ASBI implementation. However, as also mentioned by the authors, it would have been useful to have the view of front-line staff and those tasked to deliver ASBI in these challenging environments. Their views are crucial if we want to successfully implement ASBI.

In conclusion, the study provides the first and fairly good overview on the level of ASBI implementation in EDs and would be a useful benchmark with which to assess future changes in implementing ASBI in these challenging settings.

Competing interests None.

\section{References}

1. Raistrick D, Heather N, Godfrey C. Review of the Effectiveness of Treatment for Alcohol Problems. London: National Treatment Agency, 2006.

2. D’Onofrio G, Degutis LC. Preventive care in the emergency department: screening and brief intervention for alcohol problems in the emergency department: a systematic review. Acad Emerg Med 2002;9:627-38.

3. Drummond DC, Phillips T, Coulton S, et al. Saturday Night and Sunday Morning: The 2003 Twenty-Four Hour National Survey of Alcohol-Related Attendances at Accident and Emergency Departments in England. London: HMSO, 2003.

4. Green M, Setchell J, Hames P, et al. Management of alcohol abusing patients in accident and emergency departments. $J$ R Soc Med 1993;86:393-5.

5. Coulton S, Drummond C, James D, et al.; Stepwice Research Team. Opportunistic screening for alcohol use disorders in primary care: comparative study. BMJ 2006;332:511-17. 